\title{
Eventration of Diaphragm and Edward Syndrome
}

\author{
Najia al $\mathbf{H}^{1 *}$, Attia $\mathbf{A}^{1}$, Ibraheem $\mathbf{K}^{1}$, Helal al M11, Amal Z², Neda $\mathbf{B}^{1}$ \\ and Abdarhman $\mathbf{A}^{1}$ \\ 1Maternity Childeren Hospital Makkah Al Mukarama, Kingdom of Saudi Arabia \\ ${ }^{2}$ King Faisal Specialist Hospital and Research Center, Jeddah Branch, Kingdom of \\ Saudi Arabia
}

*Corresponding author: Najia al Hojaili, NICU Consultant Maternity Children Hospital Makkah Al Mukarama, Kingdom of Saudi Arabia, Email: najoo_n1@yahoo.com

\section{Summary}

Eventration of the diaphragm is an abnormal elevation of the dome of diaphragm. It is a condition in which all or part of the diaphragm is largely composed of fibrous tissue with only a few or no interspersed muscle fibers. It can be complete or partial. Complete eventration of the right diaphragm, as seen in this adult patient, is relatively rare.

Key words: Edward Syndrom; Eventration; Diaphragm; Organomegaly; Radiologically

\section{Introduction}

Congenital diaphragmatic eventration is used when there is abnormal displacement (i.e. elevation) of part or all of an otherwise intact diaphragm into the chest cavity. This rare type of CDH occurs because in the region of eventration the diaphragm is thinner, allowing the abdominal viscera to protrude upwards.

\section{Case Report}

Full term, IUGR, Delivered normal with good Apgar score to mother gravida 3 para 2, no risk factor. No maternal diseases, mother is 30years. Baby was distressed put on NCPAP and shift to NICU.

\section{On/Examination}

Baby desmorphic with low set ears, rocker bottom Feet Vital sign Temp: 37c HR: 150 RR: 40 SAT: 92 Chest: on NCPAP, good air entry on left side and demised air entry in right side CVS: IST $+2^{\mathrm{ND}}$ heart sound normal. There is systolic murmur GIT: Soft, no organomegaly, CNS: Normal tone and reflexes, anterior fontanels are normal.

\section{Investigation}

CBC :within normal Chemistry: within normal Blood Gas Respiratory acidosis Chest X-ray show eveneration of right side of diaphragm U/S brain show absent corpus collosum. U/S Chest no pleural infusion and there was eventration of diaphragm Chromosomal analysis send Bronchoscopy done shoe there is eventration of right side of diaphragm.

\section{Course and Prognosis}

Baby admitted in NICU, PUT on CPAP, Chest x- ray showed right side eventration of diaphragm, saw by pediatric surgery said conservative treatment. Pediatric cardiology started baby on Lasix syrup as there was ASD and VSD, Small PDA. 


\section{Medical Journal of Clinical Trials \& Case Studies}

\section{Discussion}

Eventration of the diaphragm is a condition in which all or part of the diaphragm is largely composed of fibrous tissue with only a few or no interspersed muscle fibers. It is usually congenital but may be acquired. Complete eventration of diaphragm invariably occurs on the left side but partial eventration of the diaphragm occurs virtually on the right side [1]. In this case, the complete eventration of diaphragm was seen on the right side which is a rarity [2]. Eventration of diaphragm is generally asymptomatic in adults and is discovered incidentally on normal screening of chest X-ray as was in the present case. Symptoms may be present in obese patients as a result of raised intra-abdominal pressure. These symptoms, related to gastrointestinal tract, respiratory embarrassment, and rarely cardiac dysfunction, have been attributed to the anomaly [3].

Elevation of diaphragm can also be attributed to interruption of phrenic nerve by neoplasm or surgical resection. In adults it is very difficult or impossible to distinguish it from diaphragmatic paralysis. These entities can be distinguished radiologically. In adults the diagnosis of diaphragmatic eventration can usually be made on standard PA and lateral chest films. In the PA projection, the elevated diaphragm forms a round unbroken line arching from the mediastinum to the costal arch [2]. Conventional chest radiography has been found to be a useful modality for assessment of the functional status of an elevated diaphragm as the evaluation of the shape of an elevated diaphragm may preclude the need for fluoroscopic sniff test to determine diaphragmatic paralysis [4].

Fluoroscopy is considered the most reliable way to document diaphragmatic paralysis and the sniff test is necessary to confirm that abnormal hemidiaphragm excursion is due to paralysis rather than unilateral weakness. Ultrasonography can help in establishing the diagnosis of partial eventration and in distinguishing it from diaphragmatic nerve interruption [5]. The diaphragm can be seen as a continuous thin layer above the elevated abdominal viscera and on real-time ultrasound the abnormal region can be seen to move downward with the normal portion although it may show a slight lag in its inspiratory excursion [6].

The radiological sight of complete eventration is identical to that diaphragmatic paralysis. In some cases, however, there is no way of knowing whether elevation is caused by congenital absence of muscle or by phrenic paralysis. Asymptomatic patients are managed conservatively but patients with symptoms require surgery. Paradoxical movements suggest complete paralysis and if symptomatic, is a strong indication of surgery [7]. In the present case, a diagnosis of eventration of diaphragm was made based on radiological findings Chromosomal analysis came showed Eduard syndrome, case discussed with pediatric surgery about gastrostomy tube is opinion to discharge patient on OGT Feeding [8].

\section{References}

1. Laxdale OE, McDougall H, Mellin GW (1954) Congenital eventration of the diaphragm. N Engl Med 250: 401-408.

2. Deslauries J (1998) Eventration of the diaphragm. Chest Surg Clin North Am 8: 315-330.

3. Chin EF, Lynn RB (1986) Surgery of eventration of the diaphragm. J Indian Med 84: 187.

4. Verhey PT, Gosselin MV, Primack SL, Kraemmer AC (2007) Differentiating diaphragmatic paralysis and eventration. Acad Radiol 14(4): 420-425.

5. Gierada DS, Slone RM, Fleishman MJ (1998) Imaging evaluation of the diaphragm. Chest Surg Clin N Am 8(2): 237-280.

6. Larson RK, Evans BH (1963) Eventration of the diaphragm. Am Rev Respir Dis 87: 753.

7. Fraser and Pare's diagnosis of diseases of the chest. $4^{\text {th }}$ (Edn.), pp: 2994-2995.

8. Articles from Lung India: Official Organ of Indian Chest Society are provided here courtesy of Wolters Kluwer, Medknow Publications.

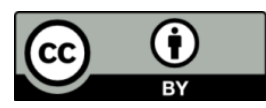

